\title{
Role of Polyelectrolyte Persistence Length in the Binding of Oppositely Charged Micelles, Dendrimers, and Protein to Chitosan and Poly(dimethyldiallyammonium chloride)
}

\author{
A. B. Kayitmazer, D. Shaw, and P. L. Dubin* \\ Department of Chemistry, IUPUI, 402 N. Blackford Street, Indianapolis, Indiana 46202 \\ Received February 15, 2005; Revised Manuscript Received April 8, 2005
}

\begin{abstract}
The effect of polyelectrolyte chain flexibility on adsorption to oppositely charged colloidal particles and its parametrization by persistence length have been investigated by comparison of PDADMAC and chitosan, polycations of equal linear charge density but of respectively low and high bare persistence lengths, relatively. Their relative affinity to (i) nonionic/anionic mixed micelles, (ii) carboxyl-terminated dendrimers, and (iii) bovine serum albumin (BSA) was studied by turbidimetric titrations. Potentiometric titrations and dynamic light scattering experiments as well as molecular modeling with SPARTAN were used to characterize and model these systems. The experimental and modeling results lead to the conclusion that while chain stiffness does influence the binding of polyelectrolytes to oppositely charged colloids, the persistence length is not necessarily an appropriate measure of chain flexibility on short length scales.
\end{abstract}

\section{Introduction}

The study of interactions between polyelectrolytes and oppositely charged small colloidal particles such as micelles, ${ }^{1}$ dendrimers,${ }^{2}$ vesicles,${ }^{3,4}$ or proteins ${ }^{5}$ provides a basis for understanding important technological and biological phenomena. Polyelectrolyte-micelle systems are directly relevant to personal care products and can also be considered as models for complexation between polyelectrolytes and other colloidal particles as long as the surfactant composition is larger than the critical micelle composition $(\mathrm{cmc})$. Studies of polyelectrolyte complexes with well-defined and symmetric dendrimers of variable high charge density are relevant to polyelectrolyte binding of small colloids. Polyelectrolyte-protein systems are more complicated due to the nonuniform shapes and charge distribution of proteins, but polyelectrolyte structural parameters can be varied systematically. Insights from such studies with model charged colloids are applicable to a number of biological phenomena, primarily interactions between proteins and biopolyelectrolytes, some examples being the regulation of DNA availability by its interaction with positively charged histones, ${ }^{6,7}$ the binding of serum proteins to hyaluronic acid in synovial fluid, and the binding of antithrombin III to heparin. Clinical and industrial applications that can benefit from insights gained with model systems include (i) enzyme immobilization, ${ }^{8}$ (ii) antigen delivery for protective immunity, ${ }^{9,10}$ (iii) design and production of biomaterials for cell micropatterning, ${ }^{11}$ (iv) biosensor design, ${ }^{12}$ (v) protein purification, ${ }^{13,14}$ and (vi) stabilization of food products. ${ }^{15}$

Extensive theoretical, simulation, and experimental studies have either anticipated or demonstrated the many parameters that affect the adsorption of polyelectrolytes onto oppositely charged colloidal surfaces. Most generally, the strength of the electrostatic interaction was found to depend on polyelectrolyte characteristics such as charge density, chain length, and chain flexibility; colloidal aspects, i.e., colloid surface charge density, size, and shape; and extensive system varia-

* Corresponding author: dubin@chem.iupui.edu. bles, i.e., temperature, $\mathrm{pH}$, and ionic strength of the medium. ${ }^{16-25}$ The magnitude of the electrostatic interaction between the polyelectrolyte and the oppositely charged colloid is found to increase with colloid surface charge density $\sigma$ and polyelectrolyte linear charge density $\xi$ and diminish with salt concentration $I$. One way to express a consensus among the numerous experimental investigations and their theoretical or simulation counterparts would thus be ${ }^{26}$

$$
\sigma_{\mathrm{c}} \xi^{a} \sim \kappa^{b}
$$

where $\sigma_{\mathrm{c}}$ is the critical colloid surface charge density at the onset of complex formation and $\kappa$ is the DebyeHuckel parameter, with $a$ and $b$ being either obtained as empirical scaling parameters or derived theoretically. Comparison between theory and experiment is of course complicated by structural features of colloid and polymer, ignored in theory. Essentially by definition, $\xi$ represents a one-dimensional array of smeared charges and thus neglects the charge heterogeneity (most obviously present in copolymers) and the location of charged groups on side chains. On the other hand, attempts have been made to take into account chain flexibility, and the goal of this paper is to consider how this property may be parametrized.

Both theories and simulations point to the effect of polyelectrolyte chain stiffness on adsorption characteristics, usually defined in terms of the persistence length, $L_{\mathrm{p}}{ }^{27-29} L_{\mathrm{p}}$ is the sum of an intrinsic contribution ( $L_{\mathrm{p}}^{\mathrm{o}}$, the bare persistence length) and an electrostatic contribution $\left(L_{\mathrm{p}}^{\mathrm{el}}\right)$. The expectation then is that $\sigma_{\mathrm{c}}$ increase with $L_{\mathrm{p}}$. Muthukumar, ${ }^{19,30}$ for example, proposed the following relationship: $\sigma_{\mathrm{c}} \sim l_{\mathrm{K}} \alpha^{2}$ at constant $I$, charge per polymer repeat unit $(q)$, colloid radius $(R)$, where $l_{\mathrm{K}}$ is the bare Kuhn length (twice the bare persistence length) and $\alpha^{2}$ is the expansion factor for the polymeric mean-square end-to-end distance. Linse and co-workers ${ }^{31,32}$ reported that a greater number of polyelectrolyte segments would reside near a spherical macroion for polyelectrolytes of low $L_{\mathrm{p}}$ in the $0-300 \mathrm{mM}$ salt concentration regime. Simulations by Stoll and co- 
workers ${ }^{26,33}$ have indicated that desorption of an oppositely charged spherical particle from a polyelectrolyte chain occurs at a critical salt concentration, which varies inversely with $L_{\mathrm{p}}$. In brief, all these studies have pointed out that flexible, i.e., low $L_{\mathrm{p}}$, polyelectrolyte chains bind more strongly to oppositely charged colloids than stiffer chains do.

Although numerous experiments on polyelectrolytecolloid systems have appeared, few studies report on the effect of chain flexibility. Binding of poly(acrylate) and poly(styrenesulfonate) to cationic micelles was considered by Macdonald and Tang, ${ }^{34}$ and the resistance of complexes to redissolution was attributed to more efficient ion pairing for more flexible polyelectrolytes. However, these two polyanions have nearly indistinguishable persistence lengths in both high salt and low salt regimes. ${ }^{35}$ Langevin and co-workers ${ }^{36}$ studied the influence of polymer backbone rigidity by comparing the complexation of cationic surfactants with a stiff chain, xanthan $\left(L_{\mathrm{p}}^{0}=5.0 \mathrm{~nm}\right.$ in water $)$, and with a flexible copolymer of $90 \%$ acrylamide (AAm) and 10\% (acrylamidomethyl)propanesulfonate (AMPS) $\left(L_{\mathrm{p}}^{0}=1.0 \mathrm{~nm}\right)$. However, binding of a single monomeric surfactant molecule to a polyelectrolyte is much more localized than the binding of a colloid. Furthermore, the nature of the product of complexation in polyelectrolytemonomeric surfactant complexes has not been wellcharacterized.

In an earlier investigation of the effect of polyelectrolyte chain stiffness on binding to oppositely charged mixed micelles, we compared two polyelectrolytes with the same linear charge density but different bare persistence lengths, namely hyaluronic acid and a copolymer of $20 \%$ AMPS and $80 \%$ AAm. ${ }^{37}$ Neither the total persistence length nor the bare persistence length could explain the divergence of their micelle binding affinities at high salt concentrations along with their convergence at low salt concentrations. To do so, it was necessary to take into account the reduction of intrapolymer electrostatic stiffening in the potential domain of the oppositely charged colloid. This led to the introduction of a modified stiffness parameter, i.e., an ionic-strength-dependent "effective" persistence length. Along similar lines, Manning ${ }^{38}$ treated the reduction of the bending energy of the polyelectrolyte complexed with a colloid of opposite charge as a consequence of the neutralization of a certain fraction of polymer charges.

There are no experimental reports on the effect of chain flexibility on adsorption of negatively charged colloids to polycations. A particular case of interest is provided by chitosan and PDADMAC, which have similar average linear charge densities but different bare persistence lengths. For chitosan, a copolymer of $(1 \rightarrow 4)$-2-amino-2-deoxy- $\beta$-D-glucan and $(1 \rightarrow 4)$-2-acetamido-2-deoxy- $\beta$-D-glucan, $L_{\mathrm{p}}^{\mathrm{o}}$ values ranging from 4.6 to $40 \mathrm{~nm}$ have been reported. ${ }^{39-44}$ The value of $6 \mathrm{~nm}$ found at $\mathrm{pH} 4.5$ with degrees of acetylation (DA) up to $55 \%$ may be considered as the most reliable one since those studies ${ }^{39}$ take into account the polydispersity of the system and the effects of excluded-volume. The value for PDADMAC is more certain: $2.5 \mathrm{~nm}$ in $0.5 \mathrm{M}$ $\mathrm{NaCl} .{ }^{45}$ The charge spacing between monomer units is $5.8 \AA(\xi \cong 1.2)$ for chitosan with $\mathrm{DA}=12 \%$ while this distance is equal to $6.2 \AA(\xi \cong 1.15)$ for PDADMAC. Thus, these two polycations are good candidates to investigate, at fixed $\xi$, the effect of polyelectrolyte chain flexibility and its parametrization by the persistence length.

Here we have studied the binding of PDADMAC and chitosan to oppositely charged (i) nonionic/anionic mixed micelles, (ii) carboxyl-terminated dendrimers, and (iii) bovine serum albumin (BSA). We note that this last case is of particular interest because of developing clinical applications of chitosan. ${ }^{46-48}$ The affinities of the polycations to micelles are defined in terms of the micelle surface charge density necessary to induce complex formation, i.e., the mole fraction of the anionic surfactant composition at the onset of binding, $Y_{\mathrm{c}}$. For the dendrimers, whose ionization states depend on $\mathrm{pH}, \mathrm{pH}_{\mathrm{c}}$ values were converted to $\alpha_{c}$, their degree of ionization at the critical conditions. Proteins also exhibit a $\mathrm{pH}_{\mathrm{c}}$, the interpretation of which is complicated by their nonuniform charge distributions. The somewhat surprising lack of correlation between colloid binding affinity and $L_{\mathrm{p}}^{\mathrm{o}}$ in all three cases is discussed in terms of the relationship between measured persistence lengths and the ability of the polymer chain to arrange its charged units proximate to the colloid charged surface.

\section{Experimental Section}

Materials and Solution Preparation. Chitosan with degrees of acetylation (DA) of $1 \%$ and $\bar{M}_{\mathrm{w}}=162 \mathrm{~K}$ was obtained from A. Domard (Université Claude Bernard-Lyon 1, Villeurbanne, France) while chitosan with DA of $12 \%\left(\bar{M}_{\mathrm{w}}=193 \mathrm{~K}\right)$ was obtained from J. Desbrières (Laboratoire de PhysicoChimie des Polymeres, Pau, France). Poly(dimethyldiallyammonium chloride) (PDADMAC) with $\bar{M}_{\mathrm{w}}$ of $219 \mathrm{~K}$ and $\bar{M}_{\mathrm{n}}$ of $141 \mathrm{~K}$ was supplied by Prof. W. Jaeger (Fraunhofer Institut, Teltow, Germany). ${ }^{49}$ Bovine serum albumin (BSA) (fatty acid free, lot 93061722) was purchased from Roche Diagnostics Corp. Sodium dodecyl sulfate (SDS), electrophoresis grade, was from Fisher Scientific. Triton X-100 (TX100) and Triton X-102 (TX102), hexaethylene glycol monododecyl ether $\left(\mathrm{C}_{12} \mathrm{E}_{6}\right)$, and octylphenol ethoxylate (OP10) were from Sigma-Aldrich, Fluka Chemie, and Nikko Chemicals, respectively. Carboxylic acidterminated cascade polymer (ZCascade: methane[4]:(3-oxo-6oxa-2-azaheptylidyne):(propanoic acid)) of generation 2 (hereafter referred to as G2) synthesized by procedures described elsewhere ${ }^{50}$ was a gift from G.R. Newkome (University of Akron). The number of terminal $\mathrm{COOH}$ groups in G2 is 36, and it has a molecular weight of $4092 . \mathrm{HCl}$ and $\mathrm{NaOH}$ standard solutions and $\mathrm{NaCl}$ from Fisher Scientific and Milli-Q water (Millipore, Milford, MA) were used throughout the work.

For all experiments, chitosan was dissolved in the required $\mathrm{NaCl}$ solution at $\mathrm{pH}$ less than 3 to ensure complete dissolution of the chitosan solution. All solutions were filtered with 0.22 $\mu \mathrm{m}$ cellulose acetate membranes (Sartorius, Viva Science Inc.).

Turbidimetric Titrations. Turbidity measurements, reported as $100-\% T(\sim$ true turbidity $(\tau)$ for $\% T>95)$, were performed at $420 \mathrm{~nm}$ using a Brinkman PC800 probe colorimeter equipped with a $2 \mathrm{~cm}$ path length fiber-optics probe. Turbidimetric "Type 1 titrations" 51 for the study of micelle/ polyelectrolyte critical binding conditions were carried out at $25 \pm 1{ }^{\circ} \mathrm{C}$ by addition of SDS to solutions of nonionic surfactant (TX100, $\mathrm{C}_{12} \mathrm{E}_{6}$, TX102, or OP10) and polymer with initial concentrations of $20 \mathrm{mM}$ and $1 \mathrm{~g} / \mathrm{L}$, respectively. The mole fraction of anionic surfactant is defined as $Y=[\mathrm{SDS}] /([\mathrm{SDS}]$ + [nonionic surfactant]), which is proportional to the mean mixed-micelle surface charge density. ${ }^{52,53}$ All turbidity values were corrected by subtracting the turbidity of a polymer-free blank.

"Type 1" titrations for protein-polycation systems were initiated at $\mathrm{pH} 2.5$ so that the net positive protein charge ensured no binding. Upon addition of $0.1 \mathrm{~N} \mathrm{NaOH}$ to solutions of $0.6 \mathrm{~g} / \mathrm{L}$ protein and $0.12 \mathrm{~g} / \mathrm{L}$ polymer (chitosan or PDAD$\mathrm{MAC}$ ) in $0.1 \mathrm{M} \mathrm{NaCl}$ at $\mathrm{pH} 4, \tau$ was measured as described above, and the $\mathrm{pH}$ was monitored with a Corning $240 \mathrm{pHmeter}$ equipped with a Beckman electrode. 


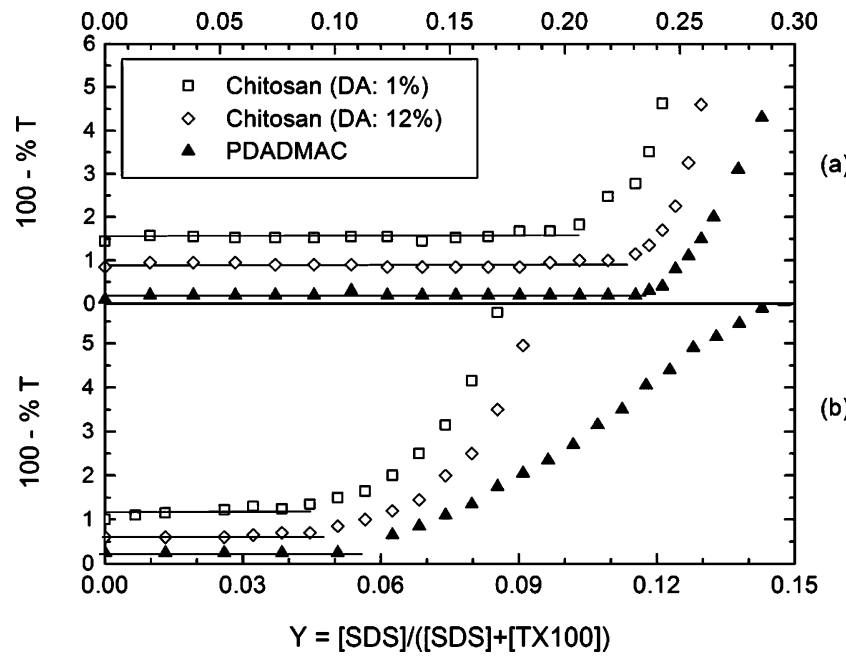

Figure 1. (a) Type 1 titrations at $I=0.4 \mathrm{M} \mathrm{NaCl}$. (b) Type 1 titrations at $I=0.05 \mathrm{M} \mathrm{NaCl}$. Horizontal lines correspond to the region in which complexation does not occur $\left(Y<Y_{\mathrm{c}}\right)$.

Potentiometric Titrations. The $\mathrm{pH}$ titrations of dendrimers were performed with a Corning $340 \mathrm{pH}$ meter equipped with a Beckman electrode and an ATC probe under $\mathrm{N}_{2}$ and magnetic stirring. Potentiometric titrations were performed by addition of $0.5 \mathrm{~N} \mathrm{HCl}$ to a $0.005 \mathrm{~g}$ dendrimer solution in $0.05 \mathrm{M} \mathrm{NaCl}$. The titration was accompanied by a dendrimerfree blank titration. The $\mathrm{pH}$ of blank and dendrimer solutions were both initially adjusted to a $\mathrm{pH}$ value of 9.97. The $\mathrm{pH}_{c}$ values obtained from the type 1 titrations of dendrimerpolycation systems were converted to critical values of the degree of ionization, $\alpha_{c}$, by fitting the $\mathrm{p} K_{\mathrm{a}}$ vs $\alpha$ plot to a line $\mathrm{e}^{54}$ for $\alpha<0.3$ and then solving the following equation (eq 2) iteratively with Excel Solver.

$$
\mathrm{p} K_{\mathrm{a}}=\mathrm{pH}+\log \left(\frac{1-\alpha}{\alpha}\right)=a \alpha+b
$$

where $a$ is the slope and $b$ is the $y$-intercept of the $\mathrm{p} K_{\mathrm{a}}$ vs $\alpha$ graph for $\alpha<0.3$.

Dynamic Light Scattering. Hydrodynamic radii of mixed micelles of OP10-SDS and TX102-SDS in $50 \mathrm{mM} \mathrm{NaCl}$ were determined by a Zetasizer Nano-ZS (Malvern Instruments).

Molecular Models. Space-filling models for PDADMAC and chitosan were created by using Spartan 'O2 SGI (Wavefunction, Inc., Irvine, CA) software. The energy minimized conformations of the polymers were determined by Monte Carlo simulations via the molecular mechanics module of the software with the "equilibrium conformer" model and Merck molecular force field (MMFF94). ${ }^{55}$

\section{Results and Discussion}

Figure 1 shows "Type 1" turbidimetric titration plots for SDS/TX100 mixed micelles with PDADMAC or chitosan samples of $1 \%$ and $12 \%$ degrees of acetylation in $0.4 \mathrm{M} \mathrm{NaCl}$, the ionic strength previously used to explore complexation in the PDADMAC and SDS/TX100 system. ${ }^{56,57}$ For comparison of binding affinities of the three polycations with SDS/TX100 micelles, the mole fraction of SDS at the point of incipient complexation, $Y_{\mathrm{c}}$, was determined from such data using a procedure

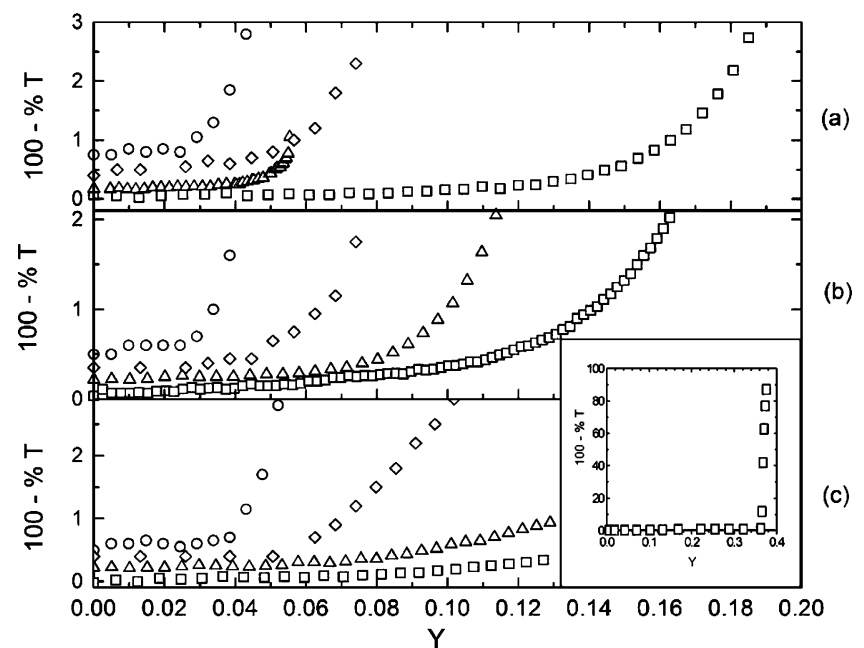

Figure 2. Type 1 titrations of (a) chitosan $(\mathrm{DA}=1 \%)$, (b) chitosan (DA $=12 \%$ ), (c) PDADMAC with mixed micelles of

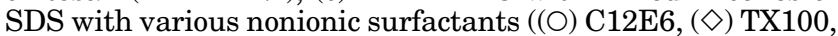
$(\triangle)$ OP10, ( $\square)$ TX102) in $0.05 \mathrm{M} \mathrm{NaCl}$. Some of the turbidity data are shifted up vertically for viewing purposes. The inset shows turbidity vs $Y$ for PDADMAC with TX102/SDS mixed micelles.

described elsewhere. ${ }^{37}$ Since $Y_{\mathrm{c}}$ is proportional to the critical micelle surface charge density $\left(\sigma_{c}\right)$, it is inversely related to the binding affinity of the polycation, in the sense that polyelectrolyte factors promoting binding, such as linear charge density, allow for binding to more weakly charged micelles. The data presented in Figure 1 show slightly higher $Y_{\mathrm{c}}$ values for PDADMAC than chitosan with DA $=12 \%$ in both 0.4 and $0.05 \mathrm{M} \mathrm{NaCl}$.

The increase in $Y_{\mathrm{c}}$ with acetylation degree (slight in the case of low salt) was expected because lower polymer charge density should go hand-in-hand with higher critical colloid surface charge density. ${ }^{25,58-60}$ However, the even larger values of $Y_{\mathrm{c}}$ for PDADMAC, presumably the more flexible polymer, were unanticipated. To evaluate the effect of persistence length more closely, we turned to variations on SDS/TX-100 with smaller micelle size, expecting that chain stiffness would inhibit binding more with a decrease in colloid radius of curvature.

In general, the effect of persistence length on polyelectrolyte-colloid binding would be expected to increase with decreasing colloid size. ${ }^{38}$ To vary micelle size, we modified the headgroup length, more bulky headgroups generally leading to smaller micelle size. ${ }^{61}$ We replaced the ethylene oxide headgroup on TX-100 (average EO length $n=9.5$ ) with EO groups of $n=10$ and $n=13$ (OP10 and TX102, respectively) and also with $n=6\left(\mathrm{C}_{12} \mathrm{E}_{6}\right.$, which lacks the benzene ring). Table 1 and Figure 2 show, as previously observed, ${ }^{62}$ that $Y_{\mathrm{c}}$ increases with $n$. As expected, smaller micelles show lower binding affinity (higher $Y_{\mathrm{c}}$ ) than larger ones. However, the effect of micelle size is complicated by additional influences of headgroup size. The EO chains should increase the distance between the polycation

Table 1. Micelle Sizes and $Y_{c}$ Values

\begin{tabular}{|c|c|c|c|c|c|c|}
\hline \multirow{2}{*}{$\begin{array}{l}\text { surfactant } \\
\text { system }\end{array}$} & \multirow[b]{2}{*}{$I(\mathrm{M})$} & \multirow[b]{2}{*}{$Y$} & \multirow[b]{2}{*}{ micelle radius $(\mathrm{nm})$} & \multicolumn{3}{|c|}{$Y_{\mathrm{c}}$} \\
\hline & & & & PDADMAC & chitosan $\mathrm{DA}=12 \%$ & chitosan DA $=1 \%$ \\
\hline $\mathrm{C}_{12} \mathrm{E}_{6}{ }^{64}$ & in $\mathrm{D}_{2} \mathrm{O}$ & 0 & $6.5 \pm 0.2$ & $0.038 \pm 0.001$ & $0.029 \pm 0.002$ & $0.026 \pm 0.002$ \\
\hline TX100-SDS 65 & 0.40 & 0.30 & 9.0 & $0.235 \pm 0.005$ & $0.227 \pm 0.005$ & $0.205 \pm 0.005$ \\
\hline TX100-SDS 66 & 0.05 & 0.05 & 4.5 & $0.056 \pm 0.005$ & $0.048 \pm 0.002$ & $0.045 \pm 0.003$ \\
\hline OP10-SDS & 0.05 & 0.07 & $4.2 \pm 0.1$ & $0.065 \pm 0.002$ & $0.062 \pm 0.002$ & $0.037 \pm 0.003$ \\
\hline TX102-SDS & 0.05 & 0.07 & $3.7 \pm 0.1$ & $0.075 \pm 0.004$ & $0.072 \pm 0.002$ & $0.080 \pm 0.005$ \\
\hline
\end{tabular}


(a)

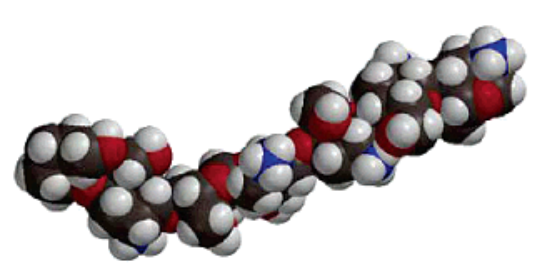

(b)

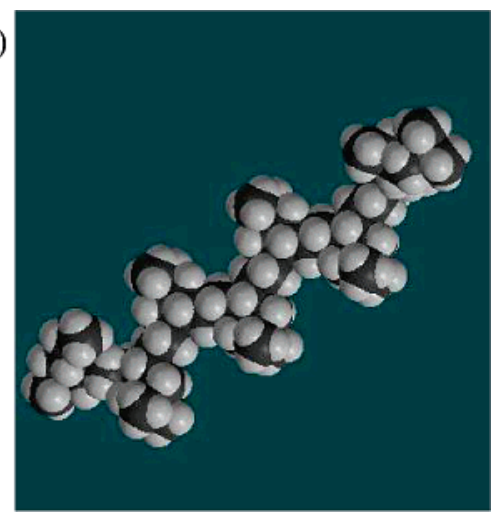

Figure 3. SPARTAN pictures of (a) chitosan and (b) PDADMAC (white: hydrogen; purple: nitrogen; gray: carbon; red: oxygen atoms).
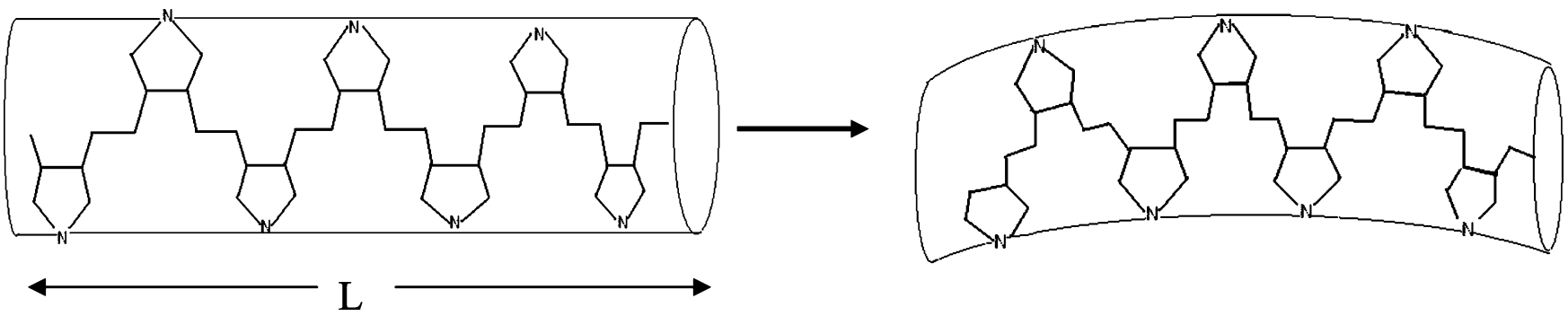

Figure 4. Cartoons of PDADMAC bending.

charge groups and the SDS sulfonate groups within the "palisade" layer, thus reducing the potential at the locus of the polycation groups, leading to an increase in the critical micelle surface charge density $\sigma_{\mathrm{c}}$ (hence, $Y_{\mathrm{c}}$ ). Furthermore, bulky nonionic headgroups could increase the average distance between SDS headgroups, reducing $\sigma$ at fixed $Y$. Thus, increases in $Y_{\mathrm{c}}$ could arise from three different effects and complicate the interpretation of nonionic headgroup substitutions.

Figure 2c also includes as an inset the turbidimetric plot for TX102/SDS with PDADMAC with an expanded turbidity scale in order to show the abrupt increase corresponding to phase separation. In general, the point of phase separation " $Y_{\phi}$ " is considerably larger than $Y_{c}$. Thus, the latter is the point of incipient soluble complex formation, as distinct from higher order aggregation or phase separation. For this reason, $Y_{\mathrm{c}}$ can be regarded as a measure of the strength of interaction between a single micelle and a corresponding length of polymer chain. A similar argument can be made for the distinction between " $\mathrm{pH}_{\phi}$ " and " $\mathrm{pH}_{\mathrm{c}}$ " for proteins, as presented below. However, it should be noted that measurements of protein-polyelectrolyte binding by capillary electrophoresis ${ }^{63}$ reveal the presence of binding on both sides of " $\mathrm{pH}_{\mathrm{c}}$ ", although the binding constant changes dramatically over a small range in $\mathrm{pH}$. The inference, regarding micelle binding, is therefore that $Y_{\mathrm{c}}$ does not represent a true phase transition but indicates rather a highly cooperative process for which the energy rapidly exceeds $k T$ with a small change in $Y$. This does not impair its effectiveness for comparisons such as those made here.

The complexity of micelle size effects does not prevent us from clearly observing that the influence of chain stiffness is counter to a priori expectations, with PDADMAC, presumably the more flexible chain, exhibiting larger $Y_{\mathrm{c}}$ values (weaker binding) than chitosan. Furthermore, the difference between $Y_{\mathrm{c}}$ for chitosan and for PDADMAC decreased for smaller micelles. More specif- ically, the results in Table 1 (excluding the single $Y_{\mathrm{c}}$ value for $1 \%$ DA chitosan and SDS/TX102) can be summarized by the following order of binding affinities (order of increasing $Y_{\mathrm{c}}$ ):

\section{$1 \%$ DA chitosan $>12 \%$ DA chitosan $>$ PDADMAC}

The stronger binding for $1 \%$ DA chitosan could be attributed to a higher charge density, but $12 \%$ DA chitosan should have the same charge density as PDADMAC, whose lower persistence length was expected to lead to stronger binding. The "effective persistence length" previously suggested ${ }^{37}$ intended to take into account the reduction of intrapolymer electrostatic stiffening in the potential domain of an oppositely charged colloid, arose from the observation that $Y_{\mathrm{c}}$ values for (stiff) hyaluronic acid and (flexible) AMPS20/ AAm80 converged at low salt and diverged at high salt. However, in this case, the similarity of the $Y_{\mathrm{c}}$ values for PDADMAC and chitosan is observed at both low and high ionic strengths. These results lead us to question the meaning of persistence length in this situation.

The persistence length, understood as the tendency of the chain to propagate in a given direction, is typically determined by light scattering and/or viscosity and is therefore defined and measured on a length scale comparable to overall chain dimensions. To consider the flexibility of the chain on the colloid length scale i.e., the micelle radius, energy-minimized conformations of the two polyelectrolytes were determined via SPARTAN. As shown in Figure 3, these SPARTAN conformations reveal the crumpled structure of PDADMAC compared to the extended structure of chitosan. This crumpled conformation of PDADMAC would lead to a decrease in the effective contour length, $L$, as indicated by both the Spartan model, and the cartoon of Figure 4. The relationship between the radius of gyration $\left(R_{\mathrm{g}}\right)$ and the persistence length for a system of monodisperse unperturbed chains is ${ }^{27}$ 


$$
R_{\mathrm{g}, \theta}^{2}=\frac{L L_{\mathrm{p}}}{3}-L_{\mathrm{p}}^{2}+\frac{2 L_{\mathrm{p}}^{3}}{L}-\frac{2 L_{\mathrm{p}}^{4}}{L}\left(1-\mathrm{e}^{-L / L_{\mathrm{p}}}\right)
$$

Inserting in eq 3 an effective contour length (smaller than the usual one based on repeat unit length and degree of polymerization) would yield, for a given measured $R_{\mathrm{g}}$, an effective persistence length larger than the nominal one. Thus, the local chain flexibility of this effective PDADMAC chain, as portrayed in Figure 4, would be less than expected from the reported persistence length based on light scattering. Put differently, the tendency of the chain to propagate in one direction per repeat unit for PDADMAC is small, but since this arises from the "folding" of the polymer chain, it does not correspond to facility in bending locally around a small colloid.

As shown in Figure 4, PDADMAC can be visualized as occupying the volume of a cylinder, on both sides of which point charges are located. If the distance between the charges on both sides of this cylinder were negligible, then one could assume the two neighboring charges to be located at the equatorial plane of the cylinder. However, the SPARTAN pictures show that the distance between two neighboring nitrogen units is around $8 \AA$, which corresponds -if we locate the cylinder at the micelle surface-to a difference in electrical potential of $10 \pm 5 \mathrm{mV}$ for two neighboring charges, one distal and the other proximate to the micelle surface. ${ }^{67,68}$ More specifically, for $\mathrm{C}_{12} \mathrm{E}_{6} / \mathrm{SDS}$ micelles in $0.05 \mathrm{M}$ $\mathrm{NaCl}$, the electrical potential drops from -11 to $-6 \mathrm{mV}$ from the micelle surface to a location $8 \AA$ away. ${ }^{68}$ Thus, when PDADMAC conforms to maximize interactions with a micelle, some of its charge groups interact more weakly with the micelle's charges; in other words, the effective linear charge density of PDADMAC is reduced relative to what might be calculated from its repeat unit structure.

As noted above, control of micelle radius by choice of nonionic surfactant leads to manifold effects. To investigate binding of a small colloidal particle, we employed the carboxylated dendrimer G2, for which $R_{\mathrm{H}}=1.7$ $\mathrm{nm} .{ }^{69}$ Here, colloid surface charge density is controlled by the charge state of the terminal carboxyl groups. The $\mathrm{pH}_{\mathrm{c}}$ values obtained as shown in Figure 5 were converted to critical values of the degree of ionization, $\alpha_{c}$, as explained in the Experimental Section (Figure 6). The results are plotted as an inset in Figure 5.

These results presented in Table 2 show that the binding affinities of these two polymers are no longer similar. At low or moderate ionic strength, the screening length is large and both distal and proximal PDADMAC charges interact with the dendrimer; then, both polymers exhibit similar binding affinity. At high salt, corresponding to a screening length of $6 \AA$, distal PDADMAC charges are at least partially screened from interaction with dendrimer, leading to a decrease in the polymer's effective charge density and a large decrease in affinity, corresponding to a value of $\alpha_{c}$ much larger than that of chitosan. Comparison to the micelle results presented above shows that the distance between polycation and colloid surface charges is clearly a more important consideration in the case of small colloids of high curvature, to which polymer configuration cannot readily adapt.

We finally consider the most complex colloidal particle, a protein with a mean hydrodynamic radius of 4 $\mathrm{nm}$, i.e., close to that of the mixed micelles, but with a

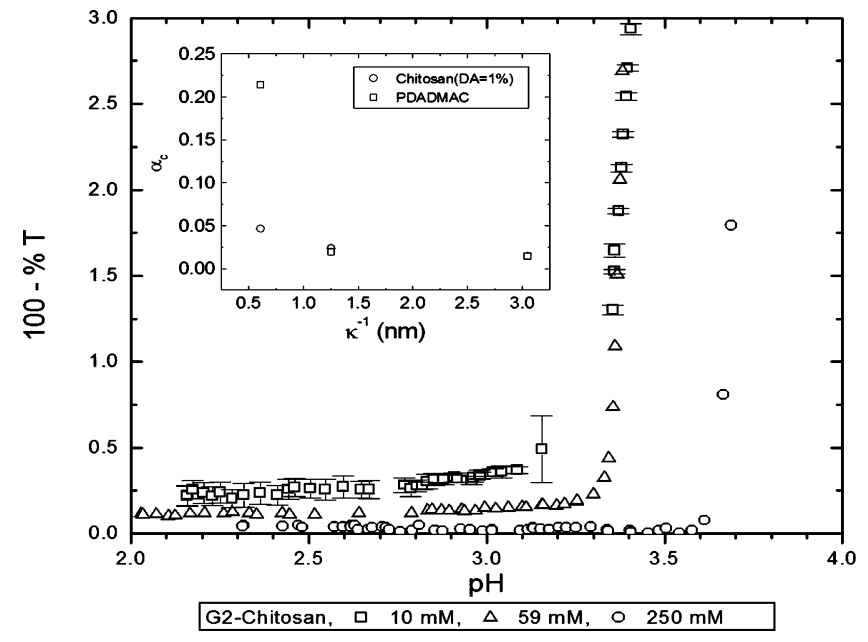

Figure 5. Type 1 titration for generation 2 carboxylated dendrimer. Inset: dependence of $\alpha_{c}$ on Debye-Hückel screening length, $\kappa^{-1}$.

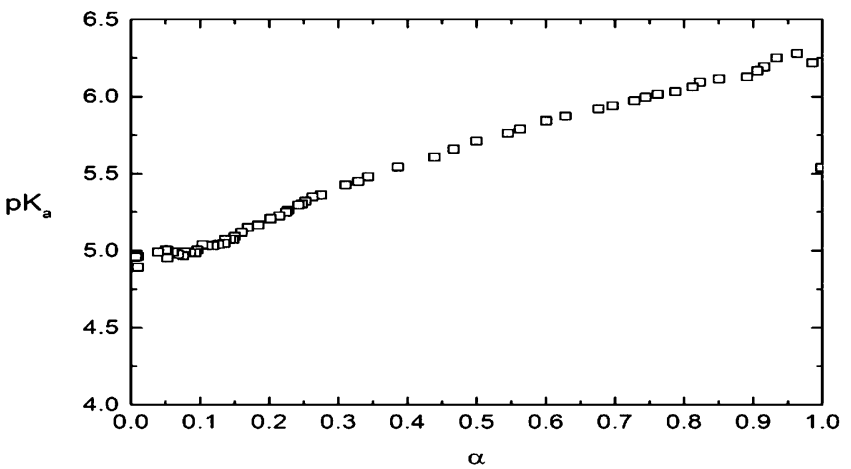

Figure 6. $\mathrm{pH}$ titration curve for $\mathrm{G} 2$ in $I=50 \mathrm{mM} \mathrm{NaCl}$.

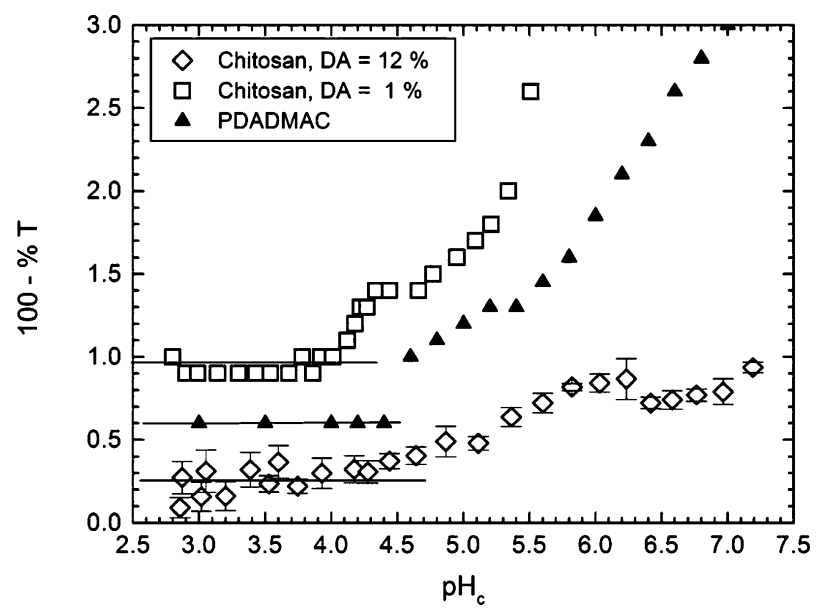

Figure 7. Turbidity vs $\mathrm{pH}$ graphs for $\mathrm{BSA}-\mathrm{PDADMAC}^{70}$ and $\mathrm{BSA}$ - chitosan interactions in $0.1 \mathrm{M} \mathrm{NaCl}$. Horizontal lines correspond to the nonbinding region. Some of the turbidity data are shifted up vertically for viewing purposes.

Table 2. Degrees of Ionization $\left(\alpha_{c}\right)$ at Critical Conditions

\begin{tabular}{ccc}
\hline & \multicolumn{2}{c}{$\alpha_{\mathrm{c}}$} \\
\cline { 2 - 3 }$I(\mathrm{mM})$ & PDADMAC & chitosan, DA $=1 \%$ \\
\hline 10 & 0.014 & 0.015 \\
59 & 0.019 & 0.023 \\
250 & 0.214 & 0.046
\end{tabular}

highly heterogeneous charge surface distribution. $\mathrm{pH}_{\mathrm{c}}$ values obtained from Figure 7 are as follows: $3.95 \pm$ 0.05 for chitosan $(\mathrm{DA}=1 \%), 4.5 \pm 0.05$ for chitosan $(\mathrm{DA}$ $=12 \%)$, and $4.5 \pm 0.15$ for PDADMAC. Thus, we can 
conclude that the $\mathrm{pH}_{\mathrm{c}}$ values are very close as in the case for micelles. However, in contrast to the micelle case, the protein charge distribution is highly asymmetric, i.e., disturbed by charge patches. For this reason, binding of these polycations to BSA occurs at $\mathrm{pH}<\mathrm{pI}$, when the protein net charge is positive. At these conditions, an additional complexity that arises is that optimal binding occurs when the polycation not only interacts with the negative patch of the protein but also avoids the surrounding positive regions; these regions can be visualized by electrostatic protein modeling. ${ }^{24}$ Chain flexibility clearly plays a more interesting and complicated role in these cases.

\section{Conclusions}

The intuitive expectation that less flexible polyelectrolyte chains encounter greater hindrance in binding to oppositely charged spherical colloidal particles is reasonable. However, the corresponding definition of stiffness - the resistance of the chain to bending to achieve proximity of opposite charges-is not necessarily correlated with the persistence length, which measures the tendency of the chain to achieve large global dimensions per unit contour length. To the extent that real polymers resemble wormlike chains, chain flexibility and persistence length would be well correlated resulting in a clear inverse dependence of binding on persistence length. Otherwise, chain flexibility with respect to binding to colloidal particles of high curvature must take into account structural details of the polymer repeat units that influence the proximity of polymer and colloid charges; conversely, low experimental values of $L_{\mathrm{p}}^{\mathrm{o}}$ can arise from locally crumpled configurations with little significance with respect to flexibility.

Acknowledgment. Support from NSF DMR0076068 is acknowledged. Prof. Alain Domard and Prof. Jacques Desbrières are thanked for kindly providing the chitosan samples.

\section{References and Notes}

(1) Polymer-Surfactant Systems; Kwak, J. C. T., Ed.; Surfactant Science Series 77; Dekker: New York, 1998; pp 482.

(2) Bielinska, A. U.; Chen, C.; Johnson, J.; Baker, J. R., Jr. Bioconjugate Chem., 1999, 10, 843-850.

(3) Menger, F. M.; Seredyuk, V. A.; Kitaeva, M. V.; Yaroslavov, A. A.; Melik-Nubarov, N. S. J. Am. Chem. Soc. 2003, 125, 2846-2847.

(4) Marques, E. F.; Regev, O.; Khan, A.; Miguel, M. d. G.; Lindman, B. Macromolecules 1999, 32, 6626-6637.

(5) Tribet, C. Complexation between amphiphilic polyelectrolytes and proteins: from necklaces to gels. In Physical Chemistry of Polyelectrolytes; Radeva, T., Ed.; Marcel Dekker: New York, 2001; Vol. 99, pp 687-741.

(6) Berg, J. M.; Tymoczko, J. L.; Stryer, L. Biochemistry, 5th ed.; W.H. Freeman and Co.: New York, 2002'.

(7) Von Hippel, P. H.; Bear, D. G.; Morgan, W. D.; McSwiggen, J. A. Annu. Rev. Biochem. 1984, 53, 389-446.

(8) Kokufuta, E. Prog. Polym. Sci. 1992, 17, 647-97.

(9) Kohn, J.; Niemi, S. M.; Albert, E. C.; Murphy, J. C.; Langer, R.; Fox, J. G. J. Immunol. Methods 1986, 95, 31-8.

(10) Andrianov, A. K.; Marin, A.; Roberts, B. E. Biomacromolecules 2005, 6, 1375-79.

(11) Kumar, G.; Wang, Y. C.; Co, C.; Ho, C.-C. Langmuir 2003 , $19,10550-10556$

(12) Chaniotakis, N. A. Anal. Bioanal. Chem. 2004, 378, 89-95.

(13) Dubin, P. L.; Gao, J.; Mattison, K. Sep. Purif. Methods 1994 $23,1-16$.

(14) Morawetz, H.; Hughes, W. L., Jr. J. Phys. Chem. 1952, 56, 64-69.

(15) Tuinier, R.; de Kruif, C. G. J. Chem. Phys. 2002, 117, 12901295.
(16) Dubin, P. L.; Oteri, R. J. Colloid Interface Sci. 1983, 95, 45361.

(17) Dubin, P. L.; Curran, M. E.; Hua, J. Langmuir 1990, 6, 707-

(18) McQuigg, D. W.; Kaplan, J. I.; Dubin, P. L. J. Phys. Chem. 1992, 96, 1973-8.

(19) von Goeler, F.; Muthukumar, M. J. Chem. Phys. 1994, 100, 7796-803.

(20) Kaibara, K.; Okazaki, T.; Bohidar, H. B.; Dubin, P. L. Biomacromolecules 2000, 1, 100-107.

(21) Wallin, T.; Linse, P. Langmuir 1996, 12, 305-14.

(22) Wallin, T.; Linse, P. J. Phys. Chem. B 1997, 101, 5506-5513.

(23) Zhulina, E. B.; Dobrynin, A. V.; Rubinstein, M. J. Phys. Chem. B 2001, 105, 8917-8930.

(24) Seyrek, E.; Dubin, P. L.; Tribet, C.; Gamble, E. A. Biomacromolecules $2003,4,273-282$.

(25) Muthukumar, M. J. Chem. Phys. 1987, 86, 7230-5.

(26) Laguecir, A.; Stoll, S.; Kirton, G.; Dubin, P. L. J. Phys. Chem. B 2003, 107, 8056-8065.

(27) Yamakawa, H. Modern Theory of Polymer Solutions; Harper and Row: New York, 1971; p 419.

(28) Yamakawa, H. Helical Wormlike Chains in Polymer Solutions; Springer: Germany, 1997; p 365.

(29) Ullner, M.; Joensson, B.; Peterson, C.; Sommelius, O.; Soederberg, B. J. Chem. Phys. 1997, 107, 1279-1287.

(30) Kong, C. Y.; Muthukumar, M. J. Chem. Phys. 1998, 109 , $1522-1527$

(31) Skepo, M.; Linse, P. Phys. Rev. E 2002, 66, 051807/1-051807/ 7.

(32) Skepö, M.; Linse, P. Macromolecules 2003, 36, 508-519.

(33) Stoll, S.; Chodanowski, P. Macromolecules 2002, 35, 95569562.

(34) Macdonald, P. M.; Tang, A., Jr. Langmuir 1997, 13, 22592265 .

(35) Tricot, M. Macromolecules 1984, 17, 1698-704.

(36) Ritacco, H.; Albouy, P.-A.; Bhattacharyya, A.; Langevin, D. Phys. Chem. Chem. Phys. 2000, 2, 5243-5251.

(37) Kayitmazer, A. B.; Seyrek, E.; Dubin, P. L.; Staggemeier, B. A. J. Phys. Chem. B 2003, 107, 8158-8165.

(38) Manning, G. S. J. Phys. Chem. B 2003, 107, 11485-11490.

(39) Berth, G.; Colfen, H.; Dautzenberg, H. Prog. Colloid Polym. Sci. 2002, 119, 50-57.

(40) Brugnerotto, J.; Desbrieres, J.; Roberts, G.; Rinaudo, M. Polymer 2001, 42, 09921-09927.

(41) Rinaudo, M.; Milas, M.; Le Dung, P. Int. J. Biol. Macromol. $1993,15,281-5$

(42) Schatz, C.; Viton, C.; Delair, T.; Pichot, C.; Domard, A. Biomacromolecules 2003, 4, 641-8.

(43) Terbojevich, M.; Carraro, C.; Cosani, A.; Marsano, E. Carbohydr. Res. 1988, 180, 73-86.

(44) Terbojevich, M. C. A.; Conio, G.; Marsano, E.; Bianchi, E. Carbohydr. Res. 1991, 209, 251-260.

(45) Dautzenberg, H.; Goernitz, E.; Jaeger, W. Macromol. Chem. Phys. 1998, 199, 1561-1571.

(46) Muzzarelli, R. A. A.; Mattioli-Belmonte, M.; Pugnaloni, A.; Biagini, G. Exs. 1999, 87, 251-264.

(47) Domard, A.; Domard, M. Polymeric Biomaterials, 2nd ed.; $2002 ; 187-212$.

(48) Rabea, E. I.; Badawy, M. E. T.; Stevens, C. V.; Smagghe, G.; Steurbaut, W. Biomacromolecules 2003, 4, 1457-65.

(49) Hahn, M.; Jaeger, W. Angew. Makromol. Chem. 1992, 198, $165-78$.

(50) Newkome, G. R.; Young, J. K.; Baker, G. R.; Potter, R. L.; Audoly, L.; Cooper, D.; Weis, C. D.; Morris, K.; Johnson, C. S., Jr. Macromolecules 1993, 26, 2394-6.

(51) Dubin, P. L.; The, S. S.; McQuigg, D. W.; Chew, C. H.; Gan, L. M. Langmuir 1989, 5, 89-95.

(52) Dubin, P. L.; Rigsbee, D. R.; McQuigg, D. W. J. Colloid Interface Sci. 1985, 105, 509-15.

(53) Dubin, P. L.; Rigsbee, D. R.; Gan, L. M.; Fallon, M. A. Macromolecules 1988, 21, 2555-9.

(54) Zhang, H. D.; P. L.; Kaplan, J.; Moorefield, C. N.; Newkome, G. R. J. Phys. Chem. B 1997, 101, 3494-3497.

(55) Halgren, T. A. J. Comput. Chem. 1996, 17, 490-519.

(56) Wang, Y.; Kimura, K.; Huang, Q.; Dubin, P. L.; Jaeger, W. Macromolecules 1999, 32, 7128-7134.

(57) Wang, Y.; Kimura, K.; Dubin, P. L.; Jaeger, W. Macromol ecules 2000, 33, 3324-3331.

(58) Wiegel, F. W. J. Phys. A: Math. Gen. 1977, 10, 299-303.

(59) Odijk, T. Macromolecules 1980, 13, 1542-6.

(60) Evers, O. A.; Fleer, G. J.; Scheutjens, J. M. H. M.; Lyklema, J. J. Colloid Interface Sci. 1986, 111, 446-54. 


\section{Kayitmazer et al.}

(61) Israelachvili, J. N. Intermolecular and Surface Forces: With Applications to Colloidal and Biological Systems; Academic Press: London, 1985; p 296.

(62) Zhang, H.; Li, Y.; Dubin, P.; Kato, T. J. Colloid Interface Sci. 1996, $183,546-551$

(63) Hattori, T.; Kimura, K.; Seyrek, E.; Dubin, P. L. Anal. Biochem. 2001, 295, 158-167.

(64) Kato, T.; Seimiya, T. J. Phys. Chem. 1986, 90, 3159-67.

(65) Li, Y.; Dubin, P. L.; Dautzenberg, H.; Lueck, U.; Hartmann, J.; Tuzar, Z. Macromolecules 1995, 28, 6795-8.

(66) Dubin, P. L.; Principi, J. M.; Smith, B. A.; Fallon, M. A. J. Colloid Interface Sci. 1989, 127, 558-65.
Macromolecules, Vol. 38, No. 12, 2005

(67) Zhang, H.; Ohbu, K.; Dubin, P. L. Langmuir 2000, 16, $9082-$ 9086.

(68) McQuigg, D. W. M.S. Thesis, Purdue University, Indianapolis, IN, 1991

(69) Young, J. K.; Baker, G. R.; Newkome, G. R.; Morris, K. F.; Johnson, C. S., Jr. Macromolecules 1994, 27, 3464-71.

(70) Mattison, K. W.; Wang, Y.; Grymonpre, K.; Dubin, P. L. Macromol. Symp. 1999, 140 (Application of Polymers in Foods), 53-76.

MA050328I 